TITLE:

\title{
Optically transparent nanofiber sheets by deposition of transparent materials: A concept for a roll-to- roll processing
}

AUTHOR(S):

Nogi, Masaya; Yano, Hiroyuki

\section{CITATION:}

Nogi, Masaya ... [et al]. Optically transparent nanofiber sheets by deposition of transparent materials: A concept for a roll-to-roll processing. Applied Physics Letters 2009, 94(23): 233117.

\section{ISSUE DATE:}

2009-06

URL:

http://hdl.handle.net/2433/84867

RIGHT:

c 2009 American Institute of Physics. 


\title{
Optically transparent nanofiber sheets by deposition of transparent materials: A concept for a roll-to-roll processing
}

\author{
Masaya Nogi（能木雅也) ${ }^{a}$ ) and Hiroyuki Yano（矢野浩之) \\ Research Institute for Sustainable Humanosphere, Kyoto University, Uji, Kyoto 611-0011, Japan
}

(Received 13 March 2009; accepted 23 May 2009; published online 12 June 2009)

\begin{abstract}
Deposition of transparent materials on cellulose nanofiber sheets enhanced the transparency of nanofiber sheets. The coated nanofiber sheets exhibited high transparency regardless of the wide distribution of refractive indexes of the coated resins, and the loss of transparency compared with the theoretical values was less than $2.5 \%$. The low coefficient of thermal expansion of the nanofiber sheets $(8.5 \mathrm{ppm} / \mathrm{K})$ was maintained after the coating. The continuous coating of functional transparent materials on the nanofiber sheets is a promising approach toward accomplishing a simple roll-to-roll manufacturing process. (c) 2009 American Institute of Physics. [DOI: $10.1063 / 1.3154547$ ]
\end{abstract}

A roll-to-roll process using flexible plastic substrate is the most anticipated to take over from conventional batch processing of glass substrates for the efficient manufacturing of various optoelectronics devices such as flat panel displays, solar cells, and radio frequency identification. ${ }^{1-3}$ However, most of the plastics have large coefficient of thermal expansion (CTE) (approximately $50 \mathrm{ppm} / \mathrm{K}$ ), which causes damage to the functional materials deposited on plastic substrates by the temperatures involved in the assembly and mounting processes, due to the mismatch between CTEs from different materials.

Recently, we have succeeded in the production of sheets of densely packed cellulose nanofibers with high modulus (13 GPa), high strength (223 MPa), minimal thermal expansion $(8.5 \mathrm{ppm} / \mathrm{K})$, and high foldability. ${ }^{4}$ When the surface of the sheet was smoothed by polishing, it became transparent. This indicates that the light scattering by the cavities in the bulk sheet is significantly suppressed because of the dense packing of cellulose nanofibers. Thus, in spite of labor intensive and unproductive polishing methods, we proposed the lamination of optically transparent plastics such as polycarbonate film onto the nanofiber sheets, and demonstrate that the lamination makes the cellulose nanofiber sheets optically transparent. ${ }^{4}$ This finding makes possible a new approach for the simple process of manufacturing optically transparent and low CTE films using multifunctional materials such as electric conductive materials (conductive polymers, indium tin oxide, graphene films, ${ }^{5}$ etc.), gas barrier films, or hard coatings. However, the deposition of transparent materials on the surface might affect its transparency due to their mismatched refractive indices (RIs).

Here we report the effects of the RIs of the depositing layer on the transparency of nanofiber sheets. The polymer coated cellulose nanofiber sheets show high optical transparency against a wide distribution of RIs of coating polymers, while maintaining low CTE of the cellulose nanofiber sheets.

Cellulose nanofibers with a width of $15 \mathrm{~nm}$ were prepared from the wood flour of Douglas fir (Pseudotsuga menziesii) using a grinder (MKCA6-3, Masuko Sangyo Co., Ltd.). ${ }^{4} \mathrm{~A}$ water suspension of nanofibers was filtered to prepare wet sheets and pressed at $200 \mathrm{kPa}$ and $120^{\circ} \mathrm{C}$ for 15

${ }^{\text {a)} E l e c t r o n i c ~ m a i l: ~ n o g i @ ~ @ i s h . k y o t o-u . a c . j p . ~}$ min. ${ }^{4}$ The resultant translucent cellulose nanofiber sheets were $40 \pm 2 \mu \mathrm{m}$ thick. Neat acrylic resin was dropped on microscope slides. The translucent sheet was sandwiched between the slides and the resins were spread on the sheets by hand pressing. The resin was immediately cured using UV curing equipment $\left(20 \mathrm{~J} / \mathrm{cm}^{2}\right.$, an F300S UV lamp system and an LC6 bench-top conveyer, Fusion UV Systems, Inc.). The polymer coated nanofiber sheets were prepared using five neat acrylic resin types with various RIs and mechanical properties (type A600, TCDDMA, KYO02, UV3000A, and UV1000A, Mitsubishi Chemical Corp., Table I). The coated nanofiber sheets were 54-60 $\mu \mathrm{m}$ thick including the resin layers of approximately $10 \mu \mathrm{m}$ on both sides and had 70 wt $\%$ fiber content on average.

The light transmittances were measured at wavelengths from 200 to $1000 \mathrm{~nm}$ using a UV-visible spectrometer with an integrating sphere $60 \mathrm{~mm}$ in diameter (U-4100, Hitachi High-Tech. Corp.). The total light transmittance and regular light transmittance were measured by placing the specimens at the entrance port and at $25 \mathrm{~cm}$ from the entrance port of the integrating sphere, respectively. To investigate the dependence of the resins' RI on the transmittances, the total and regular transmittances were compared against the RI of resins (Fig. 1). The light transmittances of the bacterial cellulose (BC) nanofiber composites impregnated with acrylic resin $(56-63 \mu \mathrm{m} \text { thickness and 56-62 wt \% fiber content })^{6}$ are also plotted in Fig. 1 to compare with the coated nanofiber sheets.

In microsized reinforcement composites the RI of reinforcements and matrix resins must be matched at least to the third decimal place to prevent light scattering. ${ }^{7}$ Contrary to this, in nanofiber composites, the RI matching to the first decimal place is enough due to the nanosize effect. ${ }^{6}$ As shown in Fig. 1(a), the BC nanofiber composites exhibit high total transmittance greater than $84.5 \%$ against a wide RI distribution, from 1.492 to 1.636 . However, the total transmittance peaks at the RI between 1.56 and 1.60, corresponding to the average RI of cellulose fiber (1.618 along the fiber and 1.544 in the transverse direction). ${ }^{8}$ The $\mathrm{BC}$ nanofiber composites were fabricated by resin impregnation into the porous nanofiber sheets with a porosity of $33 \mathrm{vol} \%$. This creates a myriad of interfaces between the matrix and the nanofibers, and causes the peak in Fig. 1(a). These results suggest that 
TABLE I. RIs, CTE, and Young's modulus of acrylic resin sheets, cellulose nanofiber sheet, and nanofiber sheets coated with various acrylic resins.

\begin{tabular}{lcccccc}
\hline \hline & \multicolumn{3}{c}{ Neat resin sheet } & & \multicolumn{2}{c}{ Coated sheet } \\
\cline { 2 - 3 } \cline { 7 - 8 } Resin type & $\mathrm{RI}$ & $\begin{array}{c}\mathrm{CTE} \\
(\mathrm{ppm} / \mathrm{K})\end{array}$ & $\begin{array}{c}E \\
(\mathrm{GPa})\end{array}$ & & $\begin{array}{c}\mathrm{CTE} \\
(\mathrm{ppm} / \mathrm{K})\end{array}$ & $\begin{array}{c}E \\
(\mathrm{GPa})\end{array}$ \\
\hline A600 & 1.492 & 214.4 & 0.018 & & $9.8(0.4)$ & $7.2(1.2)$ \\
TCDDMA & 1.532 & 86.3 & $1.59 \mathrm{GPa}$ & & $12.5(0.7)$ & $8.8(0.6)$ \\
KYO02 & 1.556 & 175.6 & 0.44 & & $13.4(1.4)$ & $8.3(1.3)$ \\
UV3000A & 1.597 & 113.8 & 0.55 & & $12.4(1.3)$ & $8.2(0.9)$ \\
UV1000A & 1.636 & 63.8 & 0.73 & & $14.9(0.7)$ & $8.0(0.8)$ \\
\hline Nanofiber sheet & $\ldots$ & $\ldots$ & $\ldots$ & & $8.9(0.4)$ & $10.7(1.5)$ \\
\hline \hline
\end{tabular}

even if $50 \mathrm{~nm}$ nanofibers are used, the RI of matrix resin should be adjusted to enhance higher optical transparency.

In contrast, there are no peaks in the total transmittances of polymer coated nanofiber sheets as shown in Fig. 1(a). Furthermore, the total transmittances of the coated nanofiber sheets decrease linearly with increasing resin RIs [Fig. 1(a)], showing a total decrease of $3.5 \%$, from $89.5 \%$ to $86.0 \%$. The theoretical total transmittances of the coated sheet were derived from the following equation: ${ }^{9}$

$$
T(\%)=\left[1-\frac{\left(n_{a}-n_{r}\right)^{2}}{\left(n_{a}+n_{r}\right)^{2}}\right]^{2} \times\left[1-\frac{\left(n_{r}-n_{c}\right)^{2}}{\left(n_{r}+n_{c}\right)^{2}}\right]^{2} \times 100,
$$

where $T$ is the theoretical total transmittances, $n_{a}$ is RI of air (1.0), $n_{r}$ is RI of coated resin, and $n_{c}$ is the average RI of
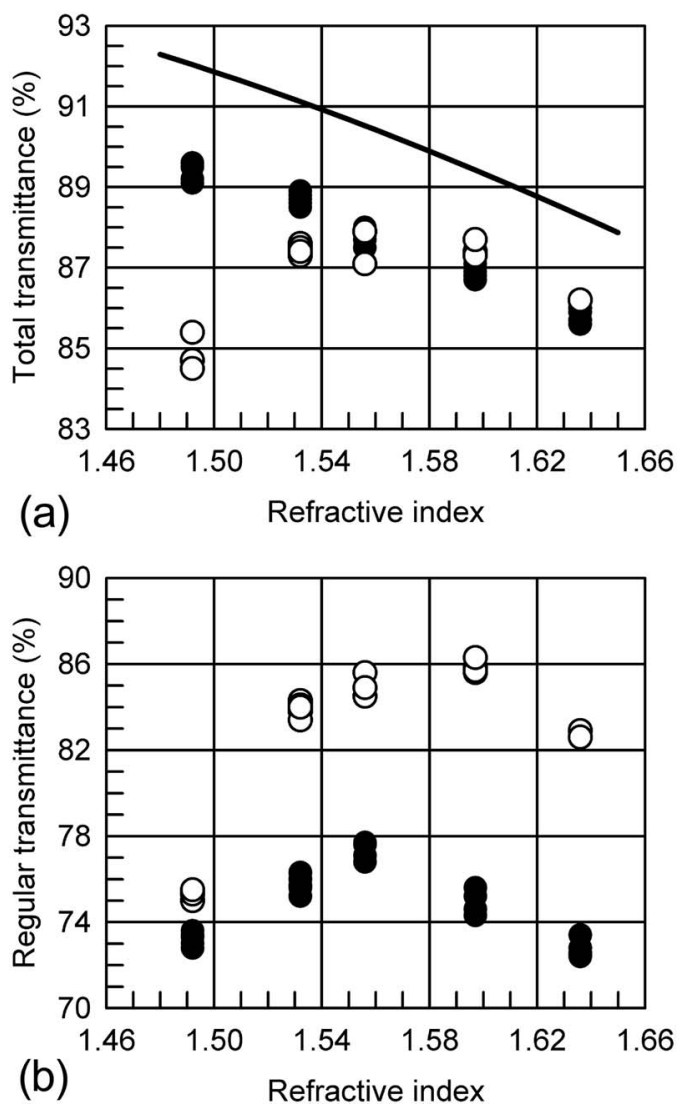

FIG. 1. (a) The total transmittance and (b) regular transmittance at $20^{\circ} \mathrm{C}$ and $590 \mathrm{~nm}$ vs the RI of resins at $20^{\circ} \mathrm{C}$ and $589.3 \mathrm{~nm}$. The filled symbols show the coated transparent nanofiber sheets $(54-60 \mu \mathrm{m}$ thickness). The open symbols show the transparent composites reinforced with BC nanofibers (56-63 $\mu \mathrm{m}$ thickness). The solid line presents the theoretical total transmittances in laminated transparent sheets. cellulose (1.581). They decrease linearly by $3.7 \%$, from $92.0 \%$ to $88.3 \%$, due to surface reflection (Fresnel's reflection) [Fig. 1(a)]. When the theoretical and experimental transmittances are compared, the loss of transparency inside the coated nanofiber sheets is less than $2.5 \%$. In addition, it is important to note that the coated nanofiber sheets exhibit much higher total transmittances as compared to $\mathrm{BC}$ nanofiber composites when the resins' RI is below 1.53. Most of the transparent plastics have lower RIs than 1.5. Thus, the nanofiber sheets coated with a great variety of plastics could attain higher optical transparency compared with nanofiber composites.

However, the regular transmittances of the coated nanofiber sheets are lower than those of BC nanofiber composites and display a gentle peak at an RI of approximately 1.56 [Fig. 1(b)]. In BC nanofiber composites, the sharp peak of regular transmittances can be explained by light scattering at the fiber/resin interfaces. In coated nanofiber sheets, the gentle peak seems to be attributed to the light scattering due to the surface roughness of cellulose nanofiber sheets since the cavities inside the nanofiber sheet are almost completely removed and thus, there are no interfaces causing light scattering inside. Hence, the effect of the surface smoothness of cellulose nanofiber sheets on regular transmittance was examined for coated nanofiber sheets (Fig. 2).

The translucent cellulose nanofiber sheet without surface smoothing showed a regular transmittance of only $6.1 \%$ at a wavelength of $590 \mathrm{~nm}$, indicative of light scattering at the sheet surface. When the translucent nanofiber sheets were

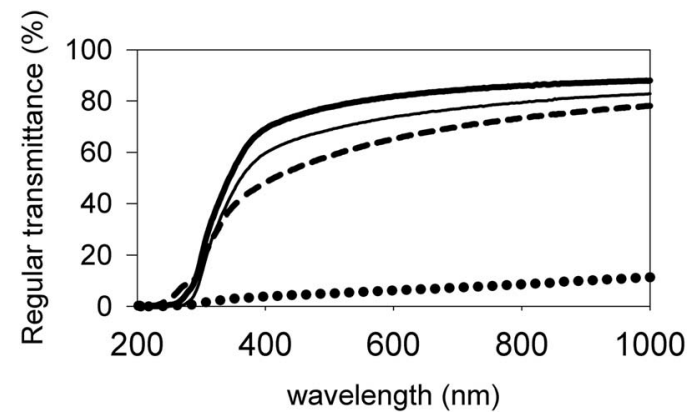

FIG. 2. The regular transmittance of various cellulose nanofiber sheets at $20{ }^{\circ} \mathrm{C}$. Dotted line shows the translucent nanofiber sheet $(40 \mu \mathrm{m}$ thickness). Broken line shows the polished nanofiber sheet without coating (40 $\mu \mathrm{m}$ thickness). Narrow line shows the nanofiber sheet coated by the acrylic resin of A600 (thickness of the nanofiber sheet before and after coating are 39 and $62 \mu \mathrm{m}$, respectively). Wide line shows the polished nanofiber sheet coated by the acrylic resin of A600 (thickness of the nanofiber sheet before and after coating are 38 and $58 \mu \mathrm{m}$, respectively). 
coated by the acrylic resin A600, the regular transmittance was $73.4 \%$ at $590 \mathrm{~nm}$ due to the elimination of surface light scattering. Alternately, when the translucent nanofiber sheets were polished by emery papers, the regular transmittance was only $64.6 \%$ at $590 \mathrm{~nm}$, which is lower than that of coated nanofiber sheet. However, when the polished sheet was coated with the acrylic resin A600, the regular transmittance reached $81.5 \%$ at $590 \mathrm{~nm}$. This represents the highest transmittance among the transparent nanofiber sheets and it is much higher than that of $\mathrm{BC}$ composites impregnated with the acrylic resin A600, which was $75.0 \%$ [Fig. 1(b)].

The total transmittances of the translucent sheet, the coated translucent sheet, the polished sheet, and the polished sheet with coating were $84.9 \%, 87.9 \%, 89.2 \%$, and $89.7 \%$, respectively. Although the regular transmittances were drastically changed by the surface treatments, the total transmittances were approximately the same. Hence, the improvement in regular transmittance accomplished by polishing indicates a reduction in haze, and suggests that the transparency of coated nanofiber sheets is influenced by their surface smoothness rather than the RIs of the coating resins.

As described above, the deposition of transparent materials creates optically transparent nanofiber sheets. However, the deposition might deteriorate the mechanical properties of the transparent sheets, such as the low thermal expansion. Thus, the Young's moduli and the CTEs of coated transparent nanofiber sheets were evaluated. As shown in Table I, cellulose nanofiber sheets show a low CTE of $8.9 \mathrm{ppm} / \mathrm{K}$ and a Young's modulus of $10.7 \mathrm{GPa}$. The transparent cellulose nanofiber sheets coated with acrylic resins have low CTEs of 9.8-14.9 ppm/K and Young's moduli of 7.2-8.8 GPa. Among those, when the acrylic resin A600 having the lowest Young's modulus was used, the coated nanofiber sheet showed the lowest CTE of $9.8 \mathrm{ppm} / \mathrm{K}$. Since the nanofiber sheets were coated with $10 \mu \mathrm{m}$ thick plastics on both sides, and soft plastics induce small amounts of thermal stress, the rigid cellulose nanofibers are able to constrain the thermal expansion of the plastics. ${ }^{10}$

The low CTE of transparent cellulose nanofiber sheets shows the advantage of the composites when compared with the CTE of the common cellulosic plastics. The CTE of cellulose acetate film (thickness $60 \mu \mathrm{m}$, degree of substitution 2.4, degree of polymerization 182, Daicel Chemical Industries, Ltd.) is $55.3 \mathrm{ppm} / \mathrm{K}$. The CTE of cellophane film (thickness $40 \mu \mathrm{m}$, PT\#300, Rengo Co., Ltd.) is $18.2 \mathrm{ppm} / \mathrm{K}$ in the flow direction and $33.6 \mathrm{ppm} / \mathrm{K}$ in the transverse direction. Moreover, it is noteworthy that the CTE of the cellulose nanofiber sheet is comparable to that of polyethylene naphthalate film $(20 \mathrm{ppm} / \mathrm{K})$ or polyimide film $(12 \mathrm{ppm} / \mathrm{K})$, which are commonly used for transparent substrates in electronic devices. ${ }^{3}$ Since the cellulose nanofiber sheets have high Young's modulus and incredibly low CTE, if the coating layers could be thinner by advanced coating or printing techniques, a much lower CTE in the coated materials would be achieved while maintaining the high optical transparency.

In conclusion, the sheet densely packed with $15 \mathrm{~nm}$ wide nanofibers exhibits high light transmittance after being coated with transparent resins that have a wide RI, while keeping a low CTE comparable to glass. Hence, the lamination or coating of optically transparent functional materials onto the cellulose nanofiber sheets can lead to a simple rollto-roll manufacturing process.

The cellulose acetate film and the cellophane film was a kind gift from Daicel Chemical Industries, Ltd. and Rengo Co., Ltd., respectively. The first author was supported by a Grant-in-Aid from Research Fellowships of the Japan Society for the Promotion of Science for Young Scientists. This work was partly supported by a Grant-In-Aid from the Integrative Industry-Academia Partnership between Kyoto University and five companies (Nippon Telegraph and Telephone Corporation, Pioneer Corporation, Hitachi, Ltd., Mitsubishi Chemical Corporation, and Rohm Co., Ltd.) and Special Coordination Funds for Promoting Science and Technology from the Ministry of Education, Culture, Sports, Science, and Technology of Japan.

\footnotetext{
${ }^{1}$ B. Zimmermann, M. Glatthaar, M. Niggemann, M. K. Riede, A. Hinsch, and A. Gombert, Sol. Energy Mater. Sol. Cells 91, 374 (2007).

${ }^{2}$ M. Pagliaro, R. Ciriminna, and G. Palmisano, ChemSusChem 1, 880 (2008).

${ }^{3}$ M. L. Chabinyc, W. S. Wong, A. C. Arias, S. Ready, R. A. Lujan, J. H. Daniel, B. Krusor, R. B. Apte, A. Salleo, and R. A. Street, Proc. IEEE 93, 1491 (2005)

${ }^{4}$ M. Nogi, S. Iwamoto, A. N. Nakagaito, and H. Yano, Adv. Mater. (Weinheim, Ger.) 21, 1595 (2009).

${ }^{5}$ K. S. Kim, Y. Zhao, H. Jang, S. Y. Lee, J. M. Kim, K. S. Kim, J. H. Ahn, P. Kim, J. Y. Choi, and B. H. Hong, Nature (London) 457, 706 (2009).

${ }^{6}$ M. Nogi, K. Handa, A. N. Nakagaito, and H. Yano, Appl. Phys. Lett. 87, 243110 (2005).

${ }^{7}$ H. Sato, H. Iba, T. Naganuma, and Y. Kagawa, Philos. Mag. B 82, 1369 (2002).

${ }^{8}$ Polymer Handbook, 4th ed., edited by J. Brandrup, E. H. Immergut, and E. A. Grulke (Wiley, New York, 1999).

${ }^{9}$ M. Born and E. Wolf, Principles of Optics, 5th ed. (Pergamon, Oxford, 1975).

${ }^{10}$ M. Nogi, and H. Yano, Adv. Mater. (Weinheim, Ger.) 20, 1849 (2008).
} 